\title{
Wave Breaking Phenomenon for DGH Equation with Strong Dissipation
}

\author{
Zhengguang Guo' ${ }^{1}$ and Min Zhao $^{2}$ \\ ${ }^{1}$ College of Mathematics and Information Science, Wenzhou University, Wenzhou, Zhejiang 325035, China \\ ${ }^{2}$ College of Life and Environmental Science, Wenzhou University, Wenzhou, Zhejiang 325035, China \\ Correspondence should be addressed to Zhengguang Guo; gzgmath@gmail.com
}

Received 7 July 2014; Revised 26 July 2014; Accepted 17 August 2014; Published 28 August 2014

Academic Editor: BoQing Dong

Copyright (c) 2014 Z. Guo and M. Zhao. This is an open access article distributed under the Creative Commons Attribution License, which permits unrestricted use, distribution, and reproduction in any medium, provided the original work is properly cited.

\begin{abstract}
The present work is mainly concerned with the Dullin-Gottwald-Holm (DGH) equation with strong dissipative term. We establish
\end{abstract} some sufficient conditions to guarantee finite time blow-up of strong solutions.

\section{Introduction}

Dullin et al. [1] derived a new equation describing the unidirectional propagation of surface waves in a shallow water regime:

$$
\begin{array}{r}
u_{t}-\alpha^{2} u_{x x t}+c_{0} u_{x}+3 u u_{x}+\gamma u_{x x x}=\alpha^{2}\left(2 u_{x} u_{x x}+u u_{x x x}\right), \\
x \in \mathbb{R}, t>0,
\end{array}
$$

where the constants $\alpha^{2}$ and $\gamma / c_{0}$ are squares of length scales and the constant $c_{0}>0$ is the critical shallow water speed for undisturbed water at rest at spatial infinity. Since this equation is derived by Dullin et al., in what follows, we call this new integrable shallow water equation (1) DGH equation.

If $\alpha=0$, (1) becomes the well-known KdV equation, whose solutions are global as long as the initial data is square integrable. This is proved by Bourgain [2]. If $\gamma=0$ and $\alpha=1$, (1) reduces to the Camassa-Holm equation, which was derived physically by Camassa and Holm in [3] by approximating directly the Hamiltonian for Euler's equations in the shallow water regime, where $u(x, t)$ represents the free surface above a flat bottom. The properties about the wellposedness, blow-up, global existence, and propagation speed for the Camassa-Holm equation have already been studied in recent papers [4-10].
It is very interesting that (1) still preserves the biHamiltonian structure and has the following two conserved quantities:

$$
\begin{gathered}
E(u)=\frac{1}{2} \int_{\mathbb{R}}\left(u^{2}+\alpha^{2} u_{x}^{2}\right) d x, \\
F(u)=\frac{1}{2} \int_{\mathbb{R}}\left(u^{3}+\alpha^{3} u u_{x}^{2}+c_{0} u^{2}-\gamma u_{x}^{2}\right) d x .
\end{gathered}
$$

Recently, in [11], local well-posedness of strong solutions to (1) was established by applying Kato's theory [12] and some sufficient conditions on the initial data were found to guarantee finite time blow-up phenomenon. Moreover, Zhou [13] found the best constants for two convolution problems on the unit circle via variational method and applied the best constants on (1) to give sufficient conditions on the initial data. Later, Zhou and Guo improved the results and got some new criteria on blow-up and then discussed the persistence properties of the strong solutions and infinite propagation speed in the recent work [14].

In general, it is quite difficult to avoid energy dissipation mechanism in the real world. Ghidaglia [15] studied the long time behavior of solutions to the weakly dissipative $\mathrm{KdV}$ equation as a finite dimensional dynamical system. Moreover, the weakly dissipative Camassa-Holm equation was investigated on the blow-up criteria and global existence 
in recent works $[16,17]$, and very related work can be found in [18]. In this work, we are interested in the following model, which can be viewed as the DGH equation with dissipation:

$$
\begin{aligned}
u_{t} & -\alpha^{2} u_{x x t}+c_{0} u_{x}+3 u u_{x}+\gamma u_{x x x}+\lambda\left(1-\alpha^{2} \partial_{x}^{2}\right) u \\
& =\alpha^{2}\left(2 u_{x} u_{x x}+u u_{x x x}\right)
\end{aligned}
$$

where $x \in \mathbb{R}, t>0, \lambda>0$, and $\lambda\left(1-\alpha^{2} \partial_{x}^{2}\right) u$ is the dissipative term which will be demonstrated when we introduce this model in the subsequent section. Indeed, (3) can be written into the following form in terms of $y=\left(1-\alpha^{2} \partial_{x}^{2}\right) u$ :

$$
\begin{array}{r}
y_{t}+(y u)_{x}+\frac{1}{2}\left(u^{2}-\alpha^{2} u_{x}^{2}\right)_{x}+c_{0}\left(u_{x}+\frac{\gamma u_{x x x}}{c_{0}}\right)+\lambda y=0, \\
x \in \mathbb{R}, t>0 .
\end{array}
$$

Set $Q=\left(1-\alpha^{2} \partial_{x}^{2}\right)^{1 / 2}$; then the operator $Q^{-2}$ can be expressed by

$$
Q^{-2} f=G * f=\int_{\mathbb{R}} G(x-y) f(y) d y,
$$

for all $f \in L^{2}(\mathbb{R})$ with $G(x)=(1 / 2 \alpha) e^{-|x| / \alpha}$. Using this identity, we can rewrite (3) as a quasilinear equation of hyperbolic type

$$
\begin{aligned}
& u_{t}+\left(u-\frac{\gamma}{\alpha^{2}}\right) u_{x}+\partial_{x} G *\left(u^{2}+\frac{\alpha^{2}}{2} u_{x}^{2}+\left(c_{0}+\frac{\gamma}{\alpha^{2}}\right) u\right) \\
& \quad+\lambda u=0
\end{aligned}
$$

where $x \in \mathbb{R}, t>0$. We find that $E(u)$ and $F(u)$ are no longer conserved for (3); this observation would make our research interesting. Note that the present dissipation model is of great importance mathematically and physically; it could be regarded as a model of a type of a certain ratedependent continuum material called a compressible secondgrade fluid [19]. We also would like to mention another dispersive DGH model. This can be achieved by replacing $\lambda\left(1-\alpha^{2} \partial_{x}^{2}\right) u$ in (3) with $\lambda\left(1-\alpha^{2} \partial_{x}^{2}\right) u_{x x x}$, and some results have been obtained by Tian's group [20]. The investigation of (3) in the periodic framework is attributed to [21]. The motivation here is to show the influence of this dissipation on the behavior of solutions, which can be illustrated by the following blow-up criteria. Precisely, we will examine the wave breaking phenomenon for the Cauchy problem and learn how parameter $\chi$ plays a role in blow-up mechanism; moreover, some comparisons will be made with the wellknown DGH equation or the Camassa-Holm equation.

In what follows, we assume that $c_{0}+\gamma / \alpha^{2}=0$ and $\alpha>0$ just for simplicity. Since $u$ is bounded by its $H^{1}$ norm, a general case with $c_{0}+\gamma / \alpha^{2} \neq 0$ does not change our results essentially, but it would lead to unnecessary technical complications. So the above equation is reduced to a simpler form:

$$
u_{t}+\left(u+c_{0}\right) u_{x}+\partial_{x} G * F(u)+\lambda u=0,
$$

where

$$
F(u)=u^{2}+\frac{\alpha^{2}}{2} u_{x}^{2}
$$

For convenience of discussion, we do scaling as follows:

$$
u(x, t) \rightarrow \widetilde{u}\left(\frac{x}{\alpha}, \frac{t}{\alpha}\right) .
$$

Therefore, (7) becomes

$$
\widetilde{u}_{t}+\left(\widetilde{u}+c_{0}\right) \tilde{u}_{x}+\partial_{x} G * F(\widetilde{u})+\lambda^{\star} \widetilde{u}=0,
$$

where

$$
\widetilde{G}(x)=\frac{1}{2} e^{-|x|}, \quad F(\widetilde{u})=\widetilde{u}^{2}+\frac{1}{2} \widetilde{u}_{x}^{2} .
$$

For concision of notations, we remove the tilde in (10) if there is no ambiguity. Then we obtain

$$
u_{t}+\left(u+c_{0}\right) u_{x}+\partial_{x} G * F(u)+\lambda u=0, \quad x \in \mathbb{R}, t>0,
$$

for some positive finite $\lambda$.

The rest of this paper is organized as follows. In Section 2, we list the local well-posedness theorem for (12) with initial datum $u_{0} \in H^{s}, s>3 / 2$, and show that the lifespan of the corresponding solution is finite if and only if its firstorder derivative blows up. In Section 3, we give some new criteria to show the generation of singularities to (12); in particular, the blow-up condition with the suitable integral form of initial momentum is involved. For simplicity, we drop $\mathbb{R}$ in our notations of function spaces if there is no ambiguity. Additionally, $\|\cdot\|_{H^{1}}$ denotes the norm of $H^{1}(\mathbb{R})$ in this paper.

\section{Preliminaries}

In this section, we make some preparations for our consideration; some results here are standard, but we still give their proofs for convenience of readers. Firstly, the local wellposedness of the Cauchy problem of (12) with initial data $u_{0} \in H^{s}$ with $s>3 / 2$ can be obtained by applying Kato's theorem [12]. More precisely, we have the following local wellposedness result.

Theorem 1. Given $u_{0}(x) \in H^{s}, s>3 / 2$, there exists $T=$ $T\left(\lambda,\left\|u_{0}\right\|_{H^{s}}\right)>0$ and a unique solution $u$ to $(12)$, such that

$$
u=u\left(\cdot, u_{0}\right) \in C\left([0, T) ; H^{s}\right) \cap C^{1}\left([0, T) ; H^{s-1}\right) .
$$

Moreover, the solution depends continuously on the initial data; that is, the mapping $u_{0} \rightarrow u\left(\cdot, u_{0}\right): H^{s} \rightarrow C\left([0, T) ; H^{s}\right) \cap$ $C^{1}\left([0, T) ; H^{s-1}\right)$ is continuous and the maximal time of existence $T>0$ is independent of $s$.

Proof. Set $A(u)=\left(u+c_{0}\right) \partial_{x} u, f(u)=-\partial_{x}\left(1-\partial_{x}^{2}\right)^{-1} F(u)-\lambda u$, $Y=H^{s}, X=H^{s-1}, s>3 / 2$, and $Q=\left(1-\partial_{x}^{2}\right)^{1 / 2}$. Applying Kato's theory for abstract quasilinear evolution equation of hyperbolic type, we obtain the local well-posedness of (12) in $H^{s}, s>3 / 2$, and $u \in C\left([0, T) ; H^{s}\right) \cap C^{1}\left([0, T) ; H^{s-1}\right)$. 
The maximal value of $T$ in Theorem 1 is usually called the lifespan of the solution. If $T<\infty$, that is, $\lim \sup _{t \rightarrow T}\|u(\cdot, t)\|_{H^{s}}=\infty$, we say the solution blows up in finite time. Next, we show that the corresponding solution blows up if and only if its first-order derivative blows up in finite time.

Theorem 2. Given $u_{0} \in H^{s}, s>3 / 2$, the solution $u=u\left(\cdot, u_{0}\right)$ of (12) blows up in finite time $T<+\infty$ if and only if

$$
\liminf _{t \rightarrow T}\left\{\inf _{x \in \mathbb{R}}\left[u_{x}(x, t)\right]\right\}=-\infty .
$$

Proof. We first assume that $u_{0} \in H^{s}$ for some $s \in \mathbb{N}, s \geq 4$. Equation (12) can be written into the following form in terms of $y=\left(1-\partial_{x}^{2}\right) u$ :

$$
y_{t}+y_{x} u+2 y u_{x}+c_{0} y_{x}+\lambda y=0 .
$$

Multiplying (15) by $y=\left(1-\partial_{x}^{2}\right) u$ and integrating by parts, we have

$$
\frac{d}{d t} \int_{\mathbb{R}} y^{2} d x=2 \int_{\mathbb{R}} y y_{t} d x=-3 \int_{\mathbb{R}} y^{2} u_{x} d x-2 \lambda \int_{\mathbb{R}} y^{2} d x .
$$

Differentiating (15) with respect to $x$, multiplying the resulting equation by $y_{x}=\left(1-\partial_{x}^{2}\right) u_{x}$, and integrating by parts again, we obtain

$$
\begin{aligned}
\frac{d}{d t} \int_{\mathbb{R}} y_{x}^{2} d x= & 2 \int_{\mathbb{R}} y_{x} y_{x t} d x \\
= & -5 \int_{\mathbb{R}} u_{x} y_{x}^{2} d x+2 \int_{\mathbb{R}} u_{x} y^{2} d x \\
& -2 \lambda \int_{\mathbb{R}} y_{x}^{2} d x .
\end{aligned}
$$

Summarizing the above two equations, we obtain

$$
\begin{aligned}
\frac{d}{d t}\left(\int_{\mathbb{R}}\left(y^{2}+y_{x}^{2}\right) d x\right)= & -5 \int_{\mathbb{R}} u_{x}\left(y^{2}+y_{x}^{2}\right) d x \\
& -2 \lambda\left(\int_{\mathbb{R}}\left(y^{2}+y_{x}^{2}\right) d x\right) .
\end{aligned}
$$

If $u_{x}$ is bounded from below on $[0, T)$, for example, $u_{x} \geq$ $-C$, where $C$ is a positive constant, then we get by (18) and Gronwall's inequality

$$
\|y\|_{H^{1}}^{2} \leq \exp \{(5 C-2 \lambda) t\}\left\|y_{0}\right\|_{H^{1}}^{2} .
$$

Therefore the $H^{3}$-norm of the solution to (12) does not blow up in finite time. Furthermore, similar argument shows that the $H^{k}$-norm with $k \geq 4$ does not blow up either in finite time. Consequently, this theorem can be proved by Theorem 1 and simple density argument for all $s>3 / 2$. On the other hand, if (14) holds, by Sobolev embedding theorem, we easily know the corresponding solution blows up in finite time. on.

Next, we prove that the energy $\|u\|_{H^{1}}^{2}$ decays as time goes
Lemma 3. Let $u_{0} \in H^{1}$; then as long as the solution $u(x, t)$ given by Theorem 1 exists, for any $t \in[0, T)$, one has

$$
\|u\|_{H^{1}}^{2}=\exp (-2 \lambda t)\left\|u_{0}\right\|_{H^{1}}^{2},
$$

where the norm is defined as

$$
\|u\|_{H^{1}}^{2}=\int_{\mathbb{R}}\left(u^{2}+u_{x}^{2}\right) d x .
$$

Proof. Multiplying both sides of (15) by $u$ and integrating by parts on $\mathbb{R}$, we get

$$
\begin{gathered}
\int_{\mathbb{R}} u y_{t} d x+\int_{\mathbb{R}}(y u)_{x} u d x+\int_{\mathbb{R}} \frac{1}{2}\left(u^{2}-u_{x}^{2}\right)_{x} u d x \\
+\int_{\mathbb{R}} c_{0} y_{x} u d x+\int_{\mathbb{R}} \lambda y u d x=0 .
\end{gathered}
$$

Note that

$$
\begin{gathered}
\int_{\mathbb{R}}(y u)_{x} u d x+\int_{\mathbb{R}} \frac{1}{2}\left(u^{2}-u_{x}^{2}\right)_{x} u d x=0, \\
\int_{\mathbb{R}} c_{0} y_{x} u d x=0 .
\end{gathered}
$$

Then, we have

$$
\int_{\mathbb{R}} u\left(u_{t}-u_{x x t}\right) d x+\int_{\mathbb{R}} \lambda\left(u^{2}-u u_{x x}\right) d x=0,
$$

and hence

$$
\int_{\mathbb{R}} u u_{t} d x-\int_{\mathbb{R}} u u_{x x t} d x+\lambda \int_{\mathbb{R}} u^{2} d x-\lambda \alpha^{2} \int_{\mathbb{R}} u u_{x x} d x=0 .
$$

Thus, we easily have

$$
\int_{\mathbb{R}}\left(u u_{t}+u_{x} u_{x t}\right) d x+\lambda \int_{\mathbb{R}}\left(u^{2}+u_{x}^{2}\right) d x=0,
$$

and therefore

$$
\frac{d}{d t}\|u\|_{H^{1}}^{2}+2 \lambda\|u\|_{H^{1}}^{2}=0 .
$$

By integration from 0 to $t$, we get

$$
\|u\|_{H^{1}}^{2}=\exp (-2 \lambda t)\left\|u_{0}\right\|_{H^{1}}^{2}, \quad \text { for any } t \in[0, T) .
$$

Hence, (20) is proved.

Lemma 4 (see [22]). Let $u(x, t)$ be the solution to $(12)$ on $[0, T)$ with initial data $u_{0} \in H^{s}, s>3 / 2$, as given by Theorem 1 . Then for function $m(t):=\inf _{x \in \mathbb{R}}\left\{u_{x}(x, t)\right\}, t>0$, there exists at least one point $\xi(t) \in \mathbb{R}$ with $m(t)=u_{x}(\xi(t), t)$; the function $m(t)$ is almost everywhere differentiable on $[0 . T)$ with

$$
\frac{d m(t)}{d t}=u_{x t}(\xi(t), t), \text { a.e. on }[0, T) .
$$

The quantity $m(t)$ is often used to consider blow-up phenomenon in the following. It is easy to derive an equation for $m(t)$ from (12) as

$$
\begin{aligned}
\frac{d m(t)}{d t}= & -\frac{1}{2} m^{2}(t)+u^{2}(\xi(t), t) \\
& -G *\left(u^{2}+\frac{1}{2} u_{x}^{2}\right)(\xi(t), t)-\lambda m(t) .
\end{aligned}
$$




\section{Wave Breaking Phenomenon}

In this section, we will establish some new criteria to guarantee the formation of singularities for the corresponding solutions to (12). The first one is as follows.

Theorem 5. Assume that $u_{0} \in H^{s}, s>3 / 2$, satisfies the following condition:

$$
u_{0 x}\left(x_{0}\right)<-\lambda-\sqrt{\lambda^{2}+\frac{1}{2}\left\|u_{0}\right\|_{H^{1}}^{2}}
$$

for some $x_{0} \in \mathbb{R}$; then the corresponding solution to (12) blows up in finite time.

Proof. In [13], Zhou has found the best constant in $\mathbb{R}$ which is different from the periodic case. The inequality is as follows:

$$
G *\left(u^{2}+\frac{1}{2} u_{x}^{2}\right)(x) \geq \frac{1}{2} u^{2}(x)
$$

Moreover, $1 / 2$ is the best constant obtained by $u=\eta e^{-|x-y|}$ for some $\eta, y \in \mathbb{R}$.

With this in hand, we have from (30)

$$
\frac{d m(t)}{d t} \leq-\frac{1}{2} m^{2}(t)-\lambda m(t)+\frac{1}{2} u^{2}(\xi(t), t) .
$$

For the best constant of Sobolev embedding $H^{1} \subset L^{\infty}$ in $\mathbb{R}$, we can compute it easily as follows. For any $x_{1} \in \mathbb{R}$,

$$
\begin{aligned}
u^{2}\left(x_{1}\right) & =\int_{-\infty}^{x_{1}} u u_{x} d x+\int_{x_{1}}^{\infty} u u_{x} d x \\
& \leq \frac{1}{2}\left(\int_{-\infty}^{x_{1}}\left(u^{2}+u_{x}^{2}\right) d x+\int_{x_{1}}^{\infty}\left(u^{2}+u_{x}^{2}\right) d x\right) \\
& =\frac{1}{2}\|u\|_{H^{1}}^{2}
\end{aligned}
$$

Thus, combining with (20), we have the following inequality:

$$
\|u\|_{L^{\infty}}^{2} \leq \frac{1}{2}\|u\|_{H^{1}}^{2}=\frac{\exp (-2 \lambda t)}{2}\left\|u_{0}\right\|_{H_{\alpha}^{1}}^{2} \leq \frac{1}{2}\left\|u_{0}\right\|_{H^{1}}^{2} .
$$

Moreover, it is easy to check that the equality is obtained by $u=\eta e^{-\left|x-x_{1}\right|}$ for any $\eta \in \mathbb{R}$.

Therefore, putting inequality (35) into (33), it follows that

$$
\begin{aligned}
\frac{d m(t)}{d t} & \leq-\frac{1}{2} m^{2}(t)-\lambda m(t)+\frac{1}{4}\left\|u_{0}\right\|_{H^{1}}^{2} \\
& =-\frac{1}{2}(m(t)+\lambda-A)(m(t)+\lambda+A),
\end{aligned}
$$

where $A=\sqrt{\lambda^{2}+(1 / 2)\left\|u_{0}\right\|_{H^{1}}^{2}}$. From the hypothesis, we have $m(0)<-\lambda-A$. Thus $\left.(d m(t) / d t)\right|_{t=0}<0$. By continuity with respect to $t$ of $m(t)$, we have $d m(t) / d t<0$, for any $t \in[0, T)$. Therefore, for any $t \in[0, T)$, we have $m(t)<-\lambda-A$. Solving the inequality above yields

$$
\frac{m(0)+\lambda-A}{m(0)+\lambda+A} \exp (-A t) \geq \frac{m(t)+\lambda-A}{m(t)+\lambda+A} .
$$

Therefore, there exists some $T$ satisfying

$$
T \leq \frac{1}{A} \ln \left(\frac{m(0)+\lambda-A}{m(0)+\lambda+A}\right)
$$

such that $\lim _{t \rightarrow T} m(t)=-\infty$. Hence, the corresponding solution of (12) blows up in finite time.

Next, we have blow-up criterion with the following form.

Theorem 6. Assume that $u_{0} \in H^{s}, s>3 / 2$, satisfies the following condition:

$$
\int_{\mathbb{R}} u_{0 x}^{3} d x<-\left(3 \lambda+\sqrt{9 \lambda^{2}+\frac{3}{2}\left\|u_{0}\right\|_{H^{1}}^{2}}\right)\left\|u_{0}\right\|_{H^{1}}^{2},
$$

and then the corresponding strong solution to (12) blows up in finite time.

Proof. From (12), differentiating both sides of it with respect to variable $x$, we obtain

$$
u_{x t}+u_{x}^{2}+u u_{x x}+c_{0} u_{x x}=u^{2}+\frac{1}{2} u_{x}^{2}-\lambda u_{x}-G * F(u)
$$

Then we can get

$$
u_{x t}=-\frac{1}{2} u_{x}^{2}+u^{2}-\left(u+c_{0}\right) u_{x x}-\lambda u_{x}-G *\left(u^{2}+\frac{1}{2} u_{x}^{2}\right) .
$$

Next multiplying $u_{x}^{2}$ on both sides of (41) and integrating by parts with respect to $x$, one obtains

$$
\begin{aligned}
\frac{d}{d t} \int_{\mathbb{R}} u_{x}^{3} d x= & -\frac{1}{2} \int_{\mathbb{R}} u_{x}^{4} d x+3 \int_{\mathbb{R}} u_{x}^{2} u^{2} d x \\
& -3 \int_{\mathbb{R}} u_{x}^{2} G *\left(u^{2}+\frac{1}{2} u_{x}^{2}\right) d x-3 \lambda \int_{\mathbb{R}} u_{x}^{3} d x
\end{aligned}
$$

In view of (41), we obtain the following inequality:

$$
\begin{aligned}
\frac{d}{d t} \int_{\mathbb{R}} u_{x}^{3} d x & \leq-\frac{1}{2} \int_{\mathbb{R}} u_{x}^{4} d x+\frac{3}{2} \int_{\mathbb{R}} u_{x}^{2} u^{2} d x-3 \lambda \int_{\mathbb{R}} u_{x}^{3} d x \\
& \leq-\frac{1}{2} \int_{\mathbb{R}} u_{x}^{4} d x+\frac{3}{4}\left\|u_{0}\right\|_{H^{1}}^{4}-3 \lambda \int_{\mathbb{R}} u_{x}^{3} d x
\end{aligned}
$$

By Cauchy-Schwartz inequality, we obtain

$$
\left(\int_{\mathbb{R}} u_{x}^{3} d x\right)^{3} \leq\left(\int_{\mathbb{R}} u_{x}^{4} d x\right)\left(\int_{\mathbb{R}} u_{x}^{2} d x\right)
$$

and it follows that

$$
\int_{\mathbb{R}} u_{x}^{4} d x \geq \frac{1}{\|u\|_{H^{1}}^{2}}\left(\int_{\mathbb{R}} u_{x}^{3} d x\right)^{2} \geq \frac{1}{\left\|u_{0}\right\|_{H^{1}}^{2}}\left(\int_{\mathbb{R}} u_{x}^{3} d x\right)^{2} .
$$


Therefore, putting it into (43), we get

$$
\begin{aligned}
\frac{d}{d t} \int_{\mathbb{R}} u_{x}^{3} d x< & -\frac{1}{2\left\|u_{0}\right\|_{H^{1}}^{2}}\left(\int_{\mathbb{R}} u_{x}^{3} d x\right)^{2}+\frac{3}{4}\left\|u_{0}\right\|_{H^{1}}^{4} \\
& -3 \lambda\left(\int_{\mathbb{R}} u_{x}^{3} d x\right) .
\end{aligned}
$$

Let $m(t)=\int_{\mathbb{R}} u_{x}^{3} d x$, with $m(0)$ denoting $\int_{\mathbb{R}} u_{0 x}^{3} d x$; by the above analysis, we get

$$
\begin{aligned}
\frac{d m(t)}{d t} \leq & -\frac{1}{2\left\|u_{0}\right\|_{H^{1}}^{2}} m^{2}(t)-3 \lambda m(t)+\frac{3}{4}\left\|u_{0}\right\|_{H^{1}}^{4} \\
= & -\frac{1}{2\left\|u_{0}\right\|_{H^{1}}^{2}}\left(m(t)+3 \lambda\left\|u_{0}\right\|_{H^{1}}^{2}-B\right) \\
& \times\left(m(t)+3 \lambda\left\|u_{0}\right\|_{H^{1}}^{2}+B\right),
\end{aligned}
$$

where $B=\left\|u_{0}\right\|_{H^{1}}^{2} \sqrt{9 \lambda^{2}+(3 / 2)\left\|u_{0}\right\|_{H^{1}}^{2}}$. The remaining part is very similar to the above theorem. We can deduce that there exists a time $T$ such that

$$
\lim _{t \uparrow T} \int_{\mathbb{R}} u_{x}^{3} d x=-\infty
$$

On the other hand,

$$
\int_{\mathbb{R}} u_{x}^{3} d x \geq \inf _{x \in \mathbb{R}} u_{x}(x, t) \int_{\mathbb{R}} u_{x}^{2} d x>C \inf _{x \in \mathbb{R}} u_{x}(x, t)\left\|u_{0}\right\|_{H^{1}}^{2},
$$

which shows that $\lim _{t \uparrow T} \inf _{x \in \mathbb{R}} u_{x}(x, t)=-\infty$. This completes the proof of Theorem 6 .

Remark 7. We note that if $\lambda=0$ in the above theorems, then the blow-up conditions are nothing but the ones established by Constantin and Zhou et al. for the Camassa-Holm equation or the DGH equation. We presented them here to show that the dissipation structure of the equation not only caused energy decay but also affected the wave breaking behavior although these arguments seem to be standard.

Motivated by McKean's deep observation for the Camassa-Holm equation [23], we can do a similar particle trajectory as

$$
\begin{gathered}
q_{t}(x, t)=u(q, t)+c_{0}, \quad 0<t<T, x \in \mathbb{R}, \\
q(x, 0)=x, \quad x \in \mathbb{R},
\end{gathered}
$$

where $u(x, t)$ is the corresponding strong solution to (12). Then for any fixed $t$ in its lifespan, $q(x, t)$ is a diffeomorphism of the line with

$$
q_{x}(x, t)=\exp \left(\int_{0}^{t} u_{x}(q, s) d s\right)>0, \quad q_{x}(x, 0)=1 .
$$

Moreover, one can verify the following important identity for the strong solution in its lifespan:

$$
\frac{d}{d t}\left(y(q(x, t), t) q_{x}^{2}(x, t)\right)=-\lambda y(q(x, t), t) q_{x}^{2}
$$

we get

$$
y(q(x, t), t) q_{x}^{2}(x, t)=y_{0}(x) \exp (-\lambda t)
$$

where $y(x, t)$ is defined by $y(x, t)=\left(1-\alpha^{2} \partial_{x}^{2}\right) u(x, t)$, for $t \geq 0$ in its lifespan.

From the expression of $u(x, t)$ in terms of $y(x, t)$, for all $t \in[0, T), x \in \mathbb{R}$, we can rewrite $u(x, t)$ and $u_{x}(x, t)$ as follows:

$$
u(x, t)=\frac{1}{2} e^{-x} \int_{-\infty}^{x} e^{\xi} y(\xi, t) d \xi+\frac{1}{2} e^{x} \int_{x}^{\infty} e^{-\xi} y(\xi, t) d \xi,
$$

from which we get that

$$
u_{x}(x, t)=-\frac{1}{2} e^{-x} \int_{-\infty}^{x} e^{\xi} y(\xi, t) d \xi+\frac{1}{2} e^{x} \int_{x}^{\infty} e^{-\xi} y(\xi, t) d \xi
$$

The following criterion shows that wave breaking occurs when the suitable integral form of initial momentum satisfies certain condition for all positive finite $\lambda$. This is motivated by the work in [13]. We do not have direct restrictions on initial velocity slope. Compared to the result in [13], the right-hand constants can be viewed as the up and down translation of the condition for DGH equation, so this observation itself is nontrivial. Obviously, if $\lambda=0$, then our result is valid for the Camassa-Holm equation. Namely, we have the following.

Theorem 8. Assume that $u_{0} \in H^{2}$ and there exists an $x_{0}$ such that the initial momentum $y_{0}\left(x_{0}\right)=0$. Furthermore,

$$
\begin{gathered}
\int_{-\infty}^{x_{0}} e^{\xi} y_{0}(\xi) d \xi>2 \lambda e^{x_{0}}, \\
\int_{x_{0}}^{\infty} e^{-\xi} y_{0}(\xi) d \xi<-2 \lambda e^{-x_{0}} .
\end{gathered}
$$

Then the corresponding solution $u(x, t)$ to (12) with initial data $u_{0}(x)$ blows up in finite time.

Proof. We obtain by (41) that

$$
u_{x t}+\left(u+c_{0}\right) u_{x x}+\frac{1}{2} u_{x}^{2}+G *\left(u^{2}+\frac{1}{2} u_{x}^{2}\right)+\lambda u_{x}-u^{2}=0
$$


Now we consider the problem at $\left(q\left(x_{0}, t\right), t\right)$, and then

$$
\begin{aligned}
\frac{d}{d t} u_{x}\left(q\left(x_{0}, t\right), t\right)= & u_{x t}\left(q\left(x_{0}, t\right), t\right) \\
& +u_{x x}\left(q\left(x_{0}, t\right), t\right)\left(u\left(q\left(x_{0}, t\right), t\right)+c_{0}\right) \\
= & \left(u^{2}-\frac{1}{2} u_{x}^{2}-G *\left(u^{2}+\frac{u_{x}^{2}}{2}\right)-\lambda u_{x}\right) \\
& \times\left(q\left(x_{0}, t\right), t\right) \\
\leq & \frac{1}{2} u^{2}\left(q\left(x_{0}, t\right), t\right)-\frac{1}{2} u_{x}^{2}\left(q\left(x_{0}, t\right), t\right) \\
& -\lambda u_{x}\left(q\left(x_{0}, t\right), t\right) \\
= & \frac{1}{4}\left[(u+\lambda)^{2}+(u-\lambda)^{2}-2\left(u_{x}+\lambda\right)^{2}\right] \\
& \times\left(q\left(x_{0}, t\right), t\right),
\end{aligned}
$$

where we use inequality (32).

Claim. $u_{x}\left(q\left(x_{0}, t\right), t\right)<0$ is decreasing, $(u+\lambda)^{2}\left(q\left(x_{0}, t\right), t\right)<$ $\left(u_{x}+\lambda\right)^{2}\left(q\left(x_{0}, t\right), t\right)$, and $(u-\lambda)^{2}\left(q\left(x_{0}, t\right), t\right)<\left(u_{x}+\right.$ $\lambda)^{2}\left(q\left(x_{0}, t\right), t\right)$, for $t \in[0, T)$, where $T$ is the maximal existence time of the solution.

By (54) and (55) and the assumption, we note that

$$
\begin{aligned}
& \left(u_{0}^{\prime}\left(x_{0}\right)+\lambda\right)^{2}-\left(u_{0}\left(x_{0}\right)+\lambda\right)^{2} \\
& \quad=\left(2 \lambda+e^{x_{0}} \int_{x_{0}}^{\infty} e^{-\xi} y_{0}(\xi) d \xi\right)\left(e^{-x_{0}} \int_{-\infty}^{x_{0}} e^{\xi} y_{0}(\xi) d \xi\right) \\
& \quad>0 \\
& \left(u_{0}^{\prime}\left(x_{0}\right)+\lambda\right)^{2}-\left(u_{0}\left(x_{0}\right)-\lambda\right)^{2} \\
& \quad=\left(e^{x_{0}} \int_{x_{0}}^{\infty} e^{-\xi} y_{0}(\xi) d \xi\right)\left(2 \lambda-e^{-x_{0}} \int_{-\infty}^{x_{0}} e^{\xi} y_{0}(\xi) d \xi\right)
\end{aligned}
$$$$
>0 \text {. }
$$

We prove the claim by method of contradiction. If not, there exists a $t_{0}$ such that

$$
\begin{array}{r}
(u+\lambda)^{2}\left(q\left(x_{0}, t\right), t\right)<\left(u_{x}+\lambda\right)^{2}\left(q\left(x_{0}, t\right), t\right), \\
t \in\left(0, t_{0}\right), \\
(u-\lambda)^{2}\left(q\left(x_{0}, t\right), t\right)<\left(u_{x}+\lambda\right)^{2}\left(q\left(x_{0}, t\right), t\right), \\
t \in\left(0, t_{0}\right),
\end{array}
$$

but

$$
(u+\lambda)^{2}\left(q\left(x_{0}, t_{0}\right), t_{0}\right) \geq\left(u_{x}+\lambda\right)^{2}\left(q\left(x_{0}, t_{0}\right), t_{0}\right),
$$

or

$$
(u-\lambda)^{2}\left(q\left(x_{0}, t_{0}\right), t_{0}\right) \geq\left(u_{x}+\lambda\right)^{2}\left(q\left(x_{0}, t_{0}\right), t_{0}\right) .
$$

For this purpose, we let

$$
\begin{aligned}
& M(t)=\frac{1}{2} e^{-q\left(x_{0}, t\right)} \int_{-\infty}^{q\left(x_{0}, t\right)} e^{\xi} y(\xi, t) d \xi \\
& N(t)=\frac{1}{2} e^{q\left(x_{0}, t\right)} \int_{q\left(x_{0}, t\right)}^{\infty} e^{-\xi} y(\xi, t) d \xi .
\end{aligned}
$$

First, differentiating $M(t)$, we obtain

$$
\begin{aligned}
\frac{d}{d t} M(t)= & -\frac{1}{2}\left(u\left(q\left(x_{0}, t\right), t\right)+c_{0}\right) e^{-q\left(x_{0}, t\right)} \\
& \times \int_{-\infty}^{q\left(x_{0}, t\right)} e^{\xi} y(\xi, t) d \xi \\
& +\frac{1}{2} e^{-q\left(x_{0}, t\right)} \int_{-\infty}^{q\left(x_{0}, t\right)} e^{\xi} y_{t}(\xi, t) d \xi .
\end{aligned}
$$

The first term of (64) is given by

$$
\begin{aligned}
-\frac{1}{2}( & \left.u\left(q\left(x_{0}, t\right), t\right)+c_{0}\right) e^{-q\left(x_{0}, t\right)} \int_{-\infty}^{q\left(x_{0}, t\right)} e^{\xi} y(\xi, t) d \xi \\
= & -\frac{1}{2} u\left(q\left(x_{0}, t\right), t\right) e^{-q\left(x_{0}, t\right)} \int_{-\infty}^{q\left(x_{0}, t\right)} e^{\xi} u(\xi, t) d \xi \\
& -\frac{1}{2} u^{2}\left(q\left(x_{0}, t\right), t\right)+\frac{1}{2} u\left(q\left(x_{0}, t\right), t\right) e^{-q\left(x_{0}, t\right)} \\
& \times \int_{-\infty}^{q\left(x_{0}, t\right)} e^{\xi} u(\xi, t) d \xi \\
& +\frac{1}{2} u\left(q\left(x_{0}, t\right), t\right) u_{x}\left(q\left(x_{0}, t\right), t\right) \\
& -\frac{1}{2} c_{0} e^{-q\left(x_{0}, t\right)} \int_{-\infty}^{q\left(x_{0}, t\right)} e^{\xi} y(\xi, t) d \xi \\
= & \frac{1}{2}\left(u u_{x}-u^{2}\right)\left(q\left(x_{0}, t\right), t\right) \\
& -\frac{1}{2} c_{0} e^{-q\left(x_{0}, t\right)} \int_{-\infty}^{q\left(x_{0}, t\right)} e^{\xi} y(\xi, t) d \xi .
\end{aligned}
$$

The second term of (64) is given by

$$
\begin{array}{r}
-\frac{1}{2} e^{-q\left(x_{0}, t\right)} \int_{-\infty}^{q\left(x_{0}, t\right)} e^{\xi}\left(y_{x} u+2 y u_{x}+c_{0} y_{x}+\lambda y\right) d \xi \\
=-\frac{1}{2} e^{-q\left(x_{0}, t\right)}\left(-\int_{-\infty}^{q\left(x_{0}, t\right)} e^{\xi}\left(u^{2}-u u_{x x}\right)(\xi, t) d \xi\right. \\
\left.+\left(\lambda-c_{0}\right) \int_{-\infty}^{q\left(x_{0}, t\right)} e^{\xi} y(\xi, t)\right) \\
-\frac{1}{2} e^{-q\left(x_{0}, t\right)}\left(\int_{-\infty}^{q\left(x_{0}, t\right)} e^{\xi}\left(u u_{x}-u_{x} u_{x x}\right)(\xi, t) d \xi\right)
\end{array}
$$




$$
\begin{aligned}
= & -\frac{1}{2} e^{-q\left(x_{0}, t\right)}\left(\lambda-c_{0}\right) \int_{-\infty}^{q\left(x_{0}, t\right)} e^{\xi} y(\xi, t) d \xi \\
& -\frac{1}{2}\left(u u_{x}\right)\left(q\left(x_{0}, t\right), t\right)+\frac{1}{4} u_{x}^{2}\left(q\left(x_{0}, t\right), t\right) \\
& +\frac{1}{2} e^{-q\left(x_{0}, t\right)}\left(\int_{-\infty}^{q\left(x_{0}, t\right)} e^{\xi}\left(u^{2}+\frac{1}{2} u_{x}^{2}\right)(\xi, t) d \xi\right) \\
\geq & \frac{1}{4} u_{x}^{2}\left(q\left(x_{0}, t\right), t\right)-\frac{1}{2}\left(u u_{x}\right)\left(q\left(x_{0}, t\right), t\right) \\
& +\frac{1}{4} u^{2}\left(q\left(x_{0}, t\right), t\right)-\frac{1}{2} \lambda\left(u-u_{x}\right)\left(q\left(x_{0}, t\right), t\right) \\
& +\frac{1}{2} c_{0} e^{-q\left(x_{0}, t\right)} \int_{-\infty}^{q\left(x_{0}, t\right)} e^{\xi} y(\xi, t) d \xi,
\end{aligned}
$$

where we have used $y\left(q\left(x_{0}, t\right), t\right)=0$ which can be derived from our assumption $y_{0}\left(x_{0}\right)=0$ and (4). Moreover, the following estimate is also used:

$$
\begin{aligned}
& \int_{-\infty}^{q\left(x_{0}, t\right)} e^{\xi}\left(u^{2}+u_{x}^{2}\right)(\xi, t) d \xi \\
& \quad \geq 2 \int_{-\infty}^{q\left(x_{0}, t\right)} e^{\xi}\left(u u_{x}\right)(\xi, t) d \xi \\
& \quad=e^{q\left(x_{0}, t\right)} u^{2}\left(q\left(x_{0}, t\right), t\right)-\int_{-\infty}^{q\left(x_{0}, t\right)} e^{\xi} u^{2}(\xi, t) d \xi
\end{aligned}
$$

Then

$$
\int_{-\infty}^{q\left(x_{0}, t\right)} e^{\xi}\left(2 u^{2}+u_{x}^{2}\right)(\xi, t) d \xi \geq e^{q\left(x_{0}, t\right)} u^{2}\left(q\left(x_{0}, t\right), t\right) .
$$

Combining (65) and (66) together yields

$$
\begin{aligned}
\frac{d}{d t} M(t) & \geq\left(\frac{1}{4} u_{x}^{2}-\frac{1}{4} u^{2}-\frac{1}{2} \lambda\left(u-u_{x}\right)\right)\left(q\left(x_{0}, t\right), t\right) \\
& =\frac{1}{4}\left[\left(u_{x}+\lambda\right)^{2}-(u+\lambda)^{2}\right]\left(q\left(x_{0}, t\right), t\right)>0 \\
& \text { on }\left[0, t_{0}\right) .
\end{aligned}
$$

Similarly, we can get for $N(t)$ that

$$
\begin{aligned}
\frac{d}{d t} N(t) & \leq\left(-\frac{1}{4} u_{x}^{2}+\frac{1}{4} u^{2}-\frac{1}{2} \lambda\left(u_{x}+u\right)\right)\left(q\left(x_{0}, t\right), t\right) \\
& =-\frac{1}{4}\left[\left(u_{x}+\lambda\right)^{2}-(u-\lambda)^{2}\right]\left(q\left(x_{0}, t\right), t\right)<0,
\end{aligned}
$$

By continuity, we note that

$$
\begin{aligned}
& {\left[\left(u_{x}+\lambda\right)^{2}-(u+\lambda)^{2}\right]\left(q\left(x_{0}, t_{0}\right), t_{0}\right)} \\
& \quad=\left(u_{x}+u+2 \lambda\right)\left(u_{x}-u\right)\left(q\left(x_{0}, t_{0}\right), t_{0}\right) \\
& \quad=4 M\left(t_{0}\right)\left(-N\left(t_{0}\right)-\lambda\right) \geq 4 M(0)(-N(0)-\lambda)>0,
\end{aligned}
$$

which contradicts our assumption. Thus, $u_{x}\left(q\left(x_{0}, t\right), t\right)$ is strictly decreasing. On the other hand, (55) and initial assumption make $u_{x}\left(q\left(x_{0}, t\right), t\right)<0$ be obvious. Therefore, the claim holds. Now let us go back to (58)

$$
\begin{aligned}
& \frac{d}{d t} u_{x}\left(q\left(x_{0}, t\right), t\right) \\
& \quad \leq \frac{1}{4}\left[(u+\lambda)^{2}+(u-\lambda)^{2}-2\left(u_{x}+\lambda\right)^{2}\right]\left(q\left(x_{0}, t\right), t\right)
\end{aligned}
$$

and denote

$$
\phi(t)=u_{x}\left(q\left(x_{0}, t\right), t\right)+\lambda .
$$

Since $\phi(t)$ is strictly decreasing with initial assumption $\phi(0)<$ 0 , there exist a $t_{1}$ and a positive constant $C_{1}$ such that, for all $t>t_{1}$, we have

$$
\begin{gathered}
\phi(t)<-\sqrt{2 C_{1}}\left\|u_{0}\right\|_{H^{1}}<0, \\
\|u \pm \lambda\|_{L^{\infty}}^{2} \leq C_{1}\left\|u_{0}\right\|_{H^{1}}^{2} .
\end{gathered}
$$

Then (72) becomes

$$
\begin{aligned}
\frac{d \phi(t)}{d t} & \leq-\frac{1}{2} \phi^{2}(t)+\frac{1}{4}\left[(u+\lambda)^{2}+(u-\lambda)^{2}\right]\left(q\left(x_{0}, t\right), t\right) \\
& \leq-\frac{1}{2} \phi^{2}(t)+\frac{1}{2} C_{1}\|u\|_{H^{1}}^{2} \\
& \leq-\frac{1}{4} \phi^{2}(t), \quad \text { for } t>t_{1} .
\end{aligned}
$$

Solving the above inequality directly, one gets

$$
\phi(t) \leq \frac{4}{4 / \phi\left(t_{1}\right)+\left(t-t_{1}\right)} .
$$

It is easy to observe that $\phi(t) \rightarrow-\infty$ as $t$ goes to $t_{1}-4 / \phi\left(t_{1}\right)$. This fact implies that the solution does not exist globally; that is, wave breaking occurs. This completes the proof of the theorem.

\section{Conflict of Interests}

The authors declare that there is no conflict of interests regarding the publication of this paper.

\section{Acknowledgments}

This work is partially supported by ZJNSF (Grant no. LQ12A01009), the National Key Basic Research Program of China (973 Program under Grant no. 2012CB426510), and NSFC (Grant no. 11301394).

\section{References}

[1] H. R. Dullin, G. A. Gottwald, and D. D. Holm, "An integrable shallow water equation with linear and nonlinear dispersion," Physical Review Letters, vol. 87, no. 19, pp. 1945-1948, 2001. 
[2] J. Bourgain, "Fourier transform restriction phenomena for certain lattice subsets and applications to nonlinear evolution equations. II. The KdV-equation," Geometric and Functional Analysis, vol. 3, no. 3, pp. 209-262, 1993.

[3] R. Camassa and D. D. Holm, "An integrable shallow water equation with peaked solitons," Physical Review Letters, vol. 71, no. 11, pp. 1661-1664, 1993.

[4] A. Constantin, "Finite propagation speed for the CamassaHolm equation," Journal of Mathematical Physics, vol. 46, no. 2, Article ID 023506, p. 4, 2005.

[5] A. Constantin and J. Escher, "Well-posedness, global existence, and blowup phenomena for a periodic quasi-linear hyperbolic equation," Communications on Pure and Applied Mathematics, vol. 51, no. 5, pp. 475-504, 1998.

[6] A. A. Himonas, G. Misiołek, G. Ponce, and Y. Zhou, "Persistence properties and unique continuation of solutions of the Camassa-Holm equation," Communications in Mathematical Physics, vol. 271, no. 2, pp. 511-522, 2007.

[7] Z. Xin and P. Zhang, "On the weak solutions to a shallow water equation," Communications on Pure and Applied Mathematics, vol. 53, no. 11, pp. 1411-1433, 2000.

[8] Y. Zhou, "Wave breaking for a shallow water equation," Nonlinear Analysis: Theory, Methods \& Applications, vol. 57, no. 1, pp. 137-152, 2004.

[9] Y. Zhou, "Wave breaking for a periodic shallow water equation," Journal of Mathematical Analysis and Applications, vol. 290, no. 2, pp. 591-604, 2004.

[10] Z. Yin, "Well-posedness, blowup, and global existence for an integrable shallow water equation," Discrete and Continuous Dynamical Systems A, vol. 11, no. 2-3, pp. 393-411, 2004.

[11] L. Tian, G. Gui, and Y. Liu, "On the well-posedness problem and the scattering problem for the Dullin-Gottwald-Holm equation," Communications in Mathematical Physics, vol. 257, no. 3, pp. 667-701, 2005.

[12] T. Kato, "Quasi-linear equations of evolution, with applications to partial differential equations," in Spectral Theory and Differential Equations: Proceedings of the Symposium held at Dundee, Scotland, 1-19 July, 1974, W. N. Everitt, Ed., vol. 448 of Lecture Notes in Mathematics, pp. 25-70, 1975.

[13] Y. Zhou, "Blow-up of solutions to the DGH equation," Journal of Functional Analysis, vol. 250, no. 1, pp. 227-248, 2007.

[14] Y. Zhou and Z. Guo, "Blow up and propagation speed of solutions to the DGH equation," Discrete and Continuous Dynamical Systems B, vol. 12, no. 3, pp. 657-670, 2009.

[15] J. Ghidaglia, "Weakly damped forced Korteweg-de Vries equations behave as a finite-dimensional dynamical system in the long time," Journal of Differential Equations, vol. 74, no. 2, pp. 369-390, 1988.

[16] S. Wu and Z. Yin, "Blow-up, blow-up rate and decay of the solution of the weakly dissipative Camassa-Holm equation," Journal of Mathematical Physics, vol. 47, no. 1, Article ID 013504, 2006.

[17] Z. Guo, "Blow up, global existence, and infinite propagation speed for the weakly dissipative Camassa-Holm equation," Journal of Mathematical Physics, vol. 49, no. 3, Article ID 033516, 9 pages, 2008.

[18] S. Wu and Z. Yin, "Global existence and blow-up phenomena for the weakly dissipative Camassa-Holm equation," Journal of Differential Equations, vol. 246, no. 11, pp. 4309-4321, 2009.

[19] A. V. Busuioc and T. S. Ratiu, "The second grade fluid and averaged Euler equations with Navier-slip boundary conditions," Nonlinearity, vol. 16, no. 3, pp. 1119-1149, 2003.
[20] L. Tian, G. Fang, and G. Gui, "Well-posedness and blowup for an integrable shallow water equation with strong dispersive term," International Journal of Nonlinear Science, vol. 1, no. 1, pp. 3-13, 2006.

[21] Z. Guo and L. Ni, "Wave breaking for the periodic weakly dissipative Dullin-Gottwald-Holm equation," Nonlinear Analysis: Theory, Methods \& Applications, vol. 74, no. 3, pp. 965-973, 2011.

[22] A. Constantin and J. Escher, "Wave breaking for nonlinear nonlocal shallow water equations," Acta Mathematica, vol. 181, no. 2, pp. 229-243, 1998.

[23] H. P. McKean, "Breakdown of the Camassa-Holm equation," Communications on Pure and Applied Mathematics, vol. 57, no. 3, pp. 416-418, 2004. 


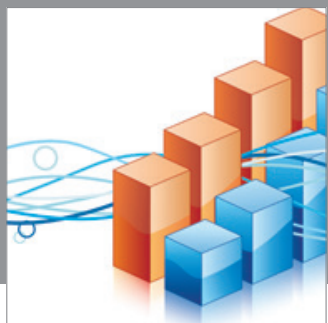

Advances in

Operations Research

mansans

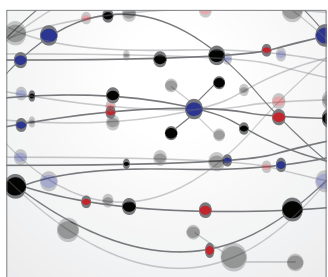

The Scientific World Journal
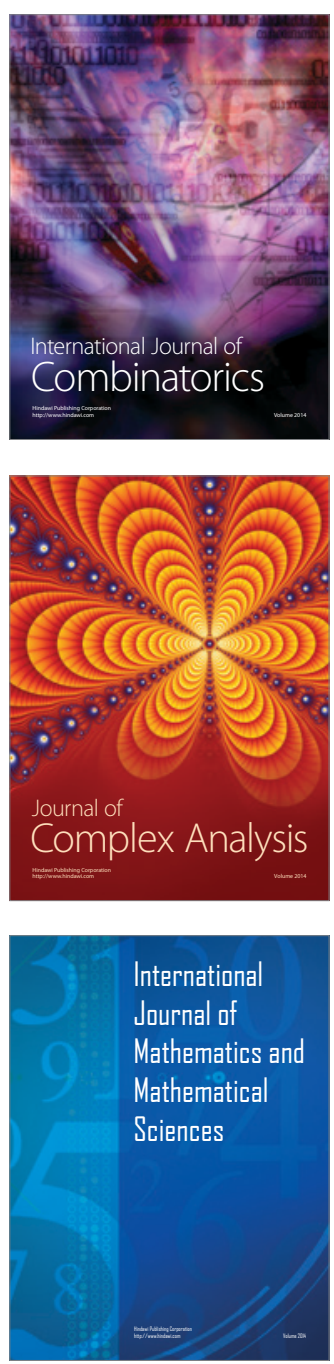
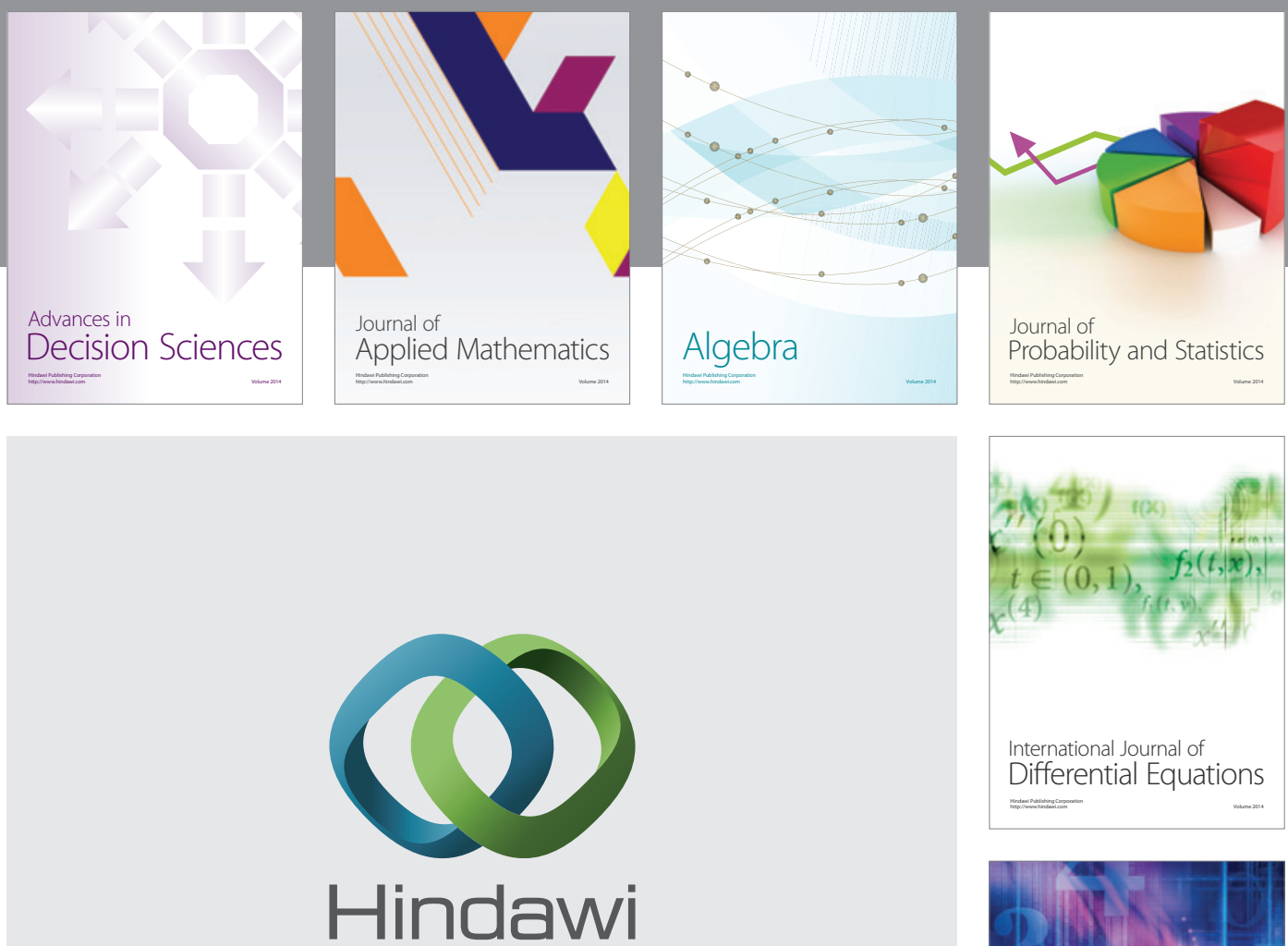

Submit your manuscripts at http://www.hindawi.com
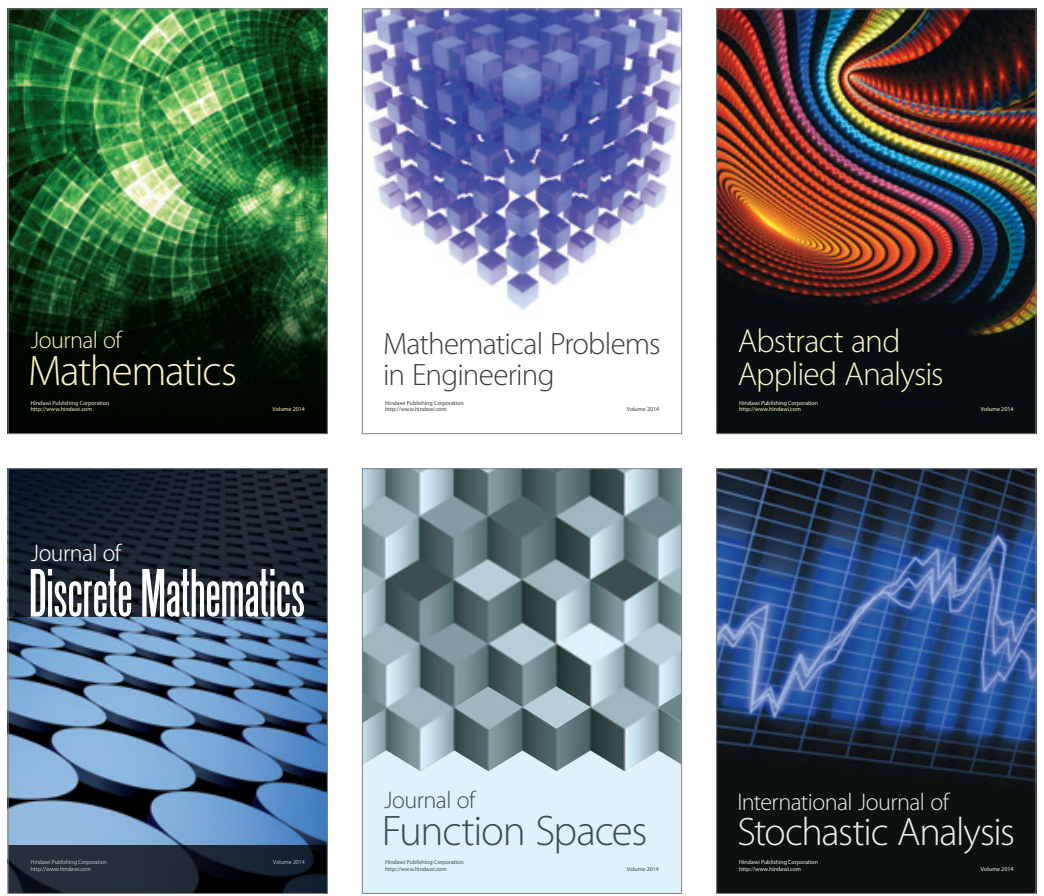

Journal of

Function Spaces

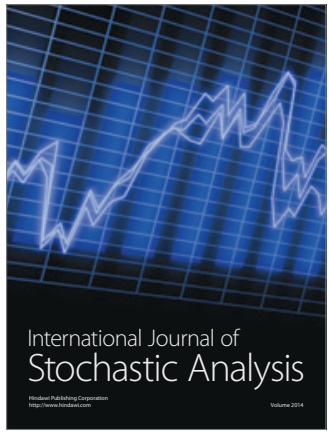

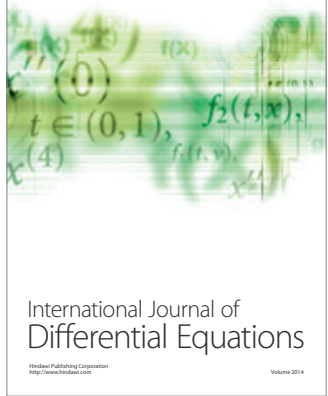
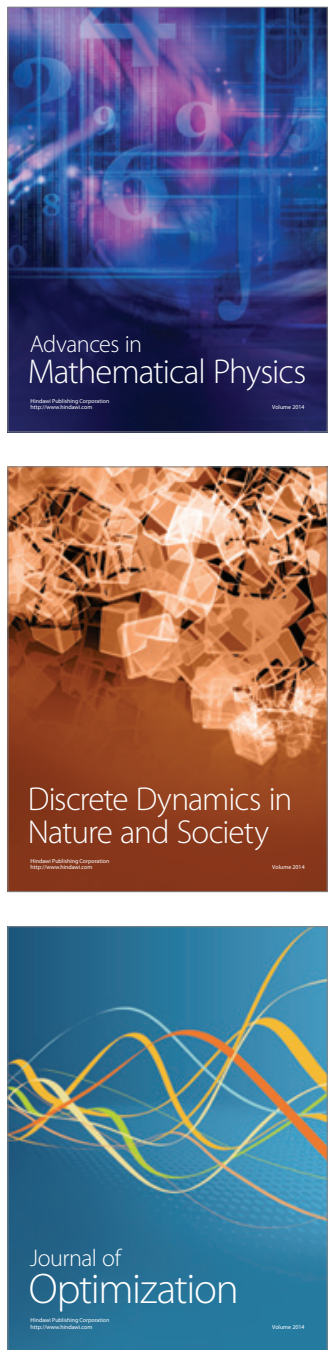